\title{
Artificial Intelligence (AI) in Video Games
}

\author{
Priya Rana \\ The Northcap University \\ Sector 23A, Gurugram, Haryana \\ 122017
}

\author{
Parthik Bhardwaj \\ The Northcap University \\ Sector 23A, Gurugram, \\ Haryana 122017
}

\author{
Jyotsna Singh, PhD \\ The Northcap University \\ Sector 23A, Gurugram, Haryana \\ 122017
}

\begin{abstract}
Computer games are an increasingly popular application for Artificial Intelligence(AI) research. This paper discusses some of the most interesting components and challenges faced by developers in designing and creation of a game based on artificial intelligence. Game AI provides players a richer gaming experience by going beyond scripted interactions, responsive interaction systems that are adaptive and intelligent.
\end{abstract}

\section{Keywords}

FSM (Finite State Machines), NPC (Non-Player Character), TBS (Turn Based Strategy), RTS (Real Time Strategy)

\section{INTRODUCTION}

The technology of computer games has been growing at a fast rate. Until recently, the technology aimed to provide realistic graphics to players. This has now been achieved. The new focused technology which is used to improve gameplay and give a realistic behavior is AI. Graphics technology makes the game look real but AI makes the game feel real and behave humanely. This paper begins with an overview of the most important game genres and role of $\mathrm{AI}$ in it. The paper briefly touches the requirement of AI in computer games and different challenges while developing AI for the computer game. Finally, some most famous Tools and Frameworks used for AI are also discussed.

Before diving into any further discussion, let it be clear that the games referred to in this paper are primarily video games that are specifically made to be played on computers. A large number of implementations of the classic games such as chess, solitaire etc are not referred here. Now we are gonna be talking about some of the interesting roles for the application of AI to these games. Following is the description of some of the more important genres of computer games on the market today.

\subsection{Games Involving Action}

These are one of the main attention drawing elements or if we say, the game genres in the market. The purpose of the game can vary from conquering an alien single-handedly to countless varieties of cannons and the fodders upon which to unleash your fury with the aid of a wide variety of exotic weaponry. The game, however, remains pretty much the same - action where the game has one aim i.e., to target and smash or shoot everything that moves. A typical action game, today takes place in a 3-d environment which is fully rendered, if viewed from a first-person perspective. For the integration of sophisticated AI, the most obvious possibilities arise in creating more and

more intelligent opponents. Finite State Machines (FSMs) are trending today. FSMs are used for determining the behavior of the player's adversaries. Despite producing a good effect, they perform poorly when confronted by situations which were not taken into 2.2 Recently, games have employed the players as the member of the squad, which will assist the player throughout the game. Such games stand out due to the impressive use of support characters and partners, eg. Tom Clancy's Rainbow Six: Rogue Spear. This is one such area which provides opportunities for the sophisticated AI.

\subsection{Games Involving Adventure}

"Adventure" was created by Don Woods and Willie Crowther in early seventies and since its creation, the adventure games have visually changed so much. However, the basics remain same for the genre of games. These games can involve the player moving around a restricted locale, a further storyline to solving puzzles and communicating and interacting with characters I the game. Nowadays, the game interacts with the users with the graphically stunning input which can be given in a variety of different ways. Otherwise, originally these messages used to be text-based, generating basic commands in English like: "eat this bun", "enter this building", "open door". The classic examples of this genre are Gabriel Knight series, Monkey Island. Adventure games have two basic and interesting AI applications that are NPCs which are more realistic and therefore, engage its audience, maintaining the consistency in dynamic story-lines.

\subsection{Games Involving Role Playing}

Oftenly, these games are seen as an extension of the adventure game style. Basically, the roleplaying games (RPGs) were developed using the concept of the popular game "Dungeons \& Dragons" which was a paper-based game that originated in the 1970's. These games have evolved so much over the past, from being text-based to the beautifully organized game which involved games available on market today. The scope involved in the game differentiates between RPGs and other adventure games. The former takes place in larger worlds and the player has been provided with more freedom in terms of exploring the environment accordingly. Also, RPGs are quite complex if compared with other adventure games as they offer same challenges to AI as any other adventure game would do. What makes it more complex is the freedom provided to the player to explore the environment on its own. Apart from this, the bigger challenge is to maintain the story consistency and also the sophistication level which is required by an RPG's Non-Player Characters.

\subsection{Games Involving Strategy}

Oftenly, these games are seen as an extension of the adventure game style. Basically, the roleplaying games (RPGs) were developed using the concept of the popular game "Dungeons \& Dragons" which was a paper-based game that originated in the 1970's. These games have evolved so much over the past, from being text-based to the beautifully organized game which involved games available on market today. The scope involved in the game differentiates between RPGs and other adventure games. The former takes place in larger worlds and the player has been provided with more freedom in terms of exploring the environment accordingly. Also, RPGs are quite 
complex if compared with other adventure games as they offer same challenges to AI as any other adventure game would do. What makes it more complex is the freedom provided to the player to explore the environment on its own. Apart from this, the bigger challenge is to maintain the story consistency and also the sophistication level which is required by an RPG's Non-Player Characters.

\subsection{Others}

Just like any attempt at categorization, all the computers can't fit well into the niches defined above. A completely original title defying categorization is released from time to time. The best example is this game having an interactive soap opera and a simulator for dollhouse called 'Sims'. This game was a huge success in 1999.

\section{REQUIREMENT FOR GAME AI}

Games can be divided into following genres: action, adventure, role-playing, strategy games, simulation, sports, and interactive drama. Among these genres, interactive drama communicates with the player which may have a strong influence on the plot. This requires AI to successfully create the game and give the player a realistic experience. The AI can mainly be applied to two different levels: 1) Individual character $\mathrm{AI}$, to produce more humanely behavior. In roleplaying games, generally, this level of AI is applied. 2) Global $\mathrm{AI}$, that looks over the game-player interaction. Real-time strategy games are generally based on this level of AI.

\section{CHALLENGES FACED WHILE COMPUTING GAME AI}

In this section, we'll briefly discuss the challenges faced by AI community while developing AI for computer games. It will give the reader an idea of what type of challenges arise to the developers.

- Unanticipated situations: It is impossible to foresee all the situations and player strategies that may encounter during gameplay. So it is difficult to build a believable behavior that will handle all the situations that may encounter.

- Knowledge engineering: Game developers gather all the knowledge about a domain to achieve a single goal, i.e. believable behavior.

- Complex decision spaces: Traditionally, game developers used handcrafted strategies to code computer games. Players easily found loopholes and exploit them. Thus, Learning techniques or higher level representations are required to deal with such complex games.

- Variability: It turns out to be tedious if the player faces the same strategies or behavior again and again. It is important to achieve variability to make game interesting and unpredictable.

- Human-like behavior: Players demands highest quality opponents which basically means a human-like opponent. It is difficult for developers to understand what human-like intelligence means to bots in games.

Each game genre has different requirements of AI. Some game genres such as interactive dramas require a mix of both levels of AI.

\section{ACADEMIC RESEARCH IN GAME}

The level of interest in AI games is growing for academic researchers. Most of the research work that has been done has come from work of military institutions. Goals are almost similar and hence there is a large crossover of techniques. For instance, SOARBOT is a project in which the agents are created based on SOAR architecture to play the 3D action game Quake. The $\mathrm{Oz}$ Project is a perfect example in the area of storytelling using computers. The project is about the application of agent-based AI technique for the task of maintaining stories which are interactive.

The Excalibur Project is another project in which agents are created to populate the virtual worlds for games.

\section{TOOLS AND FRAMEWORK}

The tools or frameworks which the scientific community is having at its disposal to test and validate the results obtained during the research. Currently, there are many tools available for free; Here's the collection of the ones which are most often used with their features which would be serving asthe reference list in the AI and video games to the researchers. ORTS game (Open Real Time Strategy) is specifically designed to be used as a research tool and get published under the GNU public license. ORTS features a protocol which is open message protocol and the client application is responsible for allowing researchers to analyze their algorithm's performance in a controlled environment with the stimulation on the server side. There is one more strategy game used widely as a research tool which is Starcraft6 featuring a library, particularly a software library called BWAPI7 which is responsible for connecting AI strategies with game engines. Another example is RoboCode8 which is a platform whose objective is developing combat robot using Java or .NET for fighting against other robots that too in real time. Planet Wars9y and ANTS10 are the games developed for the AI competitions which are hosted by Google. The former is basically a space conquest game for the users or the players who are interested in conquering all the planets on a map. On the contrary, the latter is the game for multiplayer as well in which players represent a set of ants with an objective of gathering food and conquering the rival's anthills. Eryna12 is another tool for video games which was created to support the research on AI. Being a real-time multiplayer game, it helps the players in launching games between various NPCs and then with evaluating the results. The fundamental components of this game are Game Engine following an Authoritative Server Architecture, an AI Module which can be fully customized and let the researchers develop their own NPCs and a module for generating content used in procedures which are capable of generating new maps.

SpelunkBots13, developed by Daniel Scales is a tool-set which developed from the source code of the platform game Spelunky, providing the researchers with an easy method for coding an artificial player.

\section{CONCLUSION}

A programmer simply has a task of developing an AI routine for competing against human players. Computer games do not intend at defeating the human player, but providing them an exciting playing adventure. The player would give up soon if the computer will play too strongly and in the case where the user could defeat the computer very easily, the victory won't be so sweat.

The same mission can be played, in different levels, differently in various computer games to be able to make the game enjoyable for different human players (according to the skills).

Sometimes we come across the situations where the computer is (unambiguosly) better than a human player; for example 
when we have to aim in a shooting game or in a simple situation where the multiplication of large numbers is invloved. These are such situation which requires a precise numeric computation based on a specific scheme formulated for winning. Since computers were invented purposely to solve such problems, they are strong in computational aspect, but in all the other cases humans are inevitably better than computers: comprehending and manipulation of the game; on the basis of one's feelings- making a decision, etc. are such examples where humans seem superior than machines.

Of course, as proven by many psychological and sociological experiments, human thinking cannot be considered to be optimal, but humans are the only species who have written a program that could play a game better than humans itself.

\section{REFERENCES}

[1] Research Directions for AI in Computer Games; Chris Fairclough, Michael Fagan, Brian Mac Namee, Pádraig Cunningham, Department of Computer Science, Trinity College, Dublin 2, Ireland.

[2] Artificial Intelligence for Adaptive Computer Games; Ashwin Ram, Santiago Ontãn'on, and Manish Mehta;
Cognitive Computing Lab (CCL), College of Computing, Georgia Institute of Technology, Atlanta, Georgia, USA

[3] Game Artificial Intelligence: Challenges for the Scientific Community; Raúl Lara-Cabrera, Mariela NogueiraCollazo, Carlos Cotta and Antonio J.Fernández-Leiva; Department Lenguajes y Ciencias de la Computación, ETSI Informática, University

[4] Smith, S. and Bates, J., Towards a Theory of Narrative for Interactive Fiction, CMU-CS-89121, 1989.

[5] Stout, B., Smart Moves: Intelligent Path-Finding, Game Developer Magazine, October 1996.

[6] Lester, J., and Stone, B. 1997. Increasing believability in animated pedagogical agents. In First International Conference on Autonomous Agents, 16-21.

[7] Meehan, J., The meta-novel: writing stories on the computer. University Microfilms International, 1977.

[8] Johansson, A., Dell'Acqua, P.: Emotional behavior trees. In: Computational Intelligence and Games (CIG), 2012 IEEE Conference on. pp. 355362. IEEE (2012) 